\title{
DIFFICULTIES IN THE SIMULTANEOUS MEASUREMENT \\ OF ALL STOKES PARAMETERS
}

\author{
E. WIEHR
}

Universitäts-Sternwarte, Göttingen, Germany

Telescopic phase retardation in connection with the polarizer behind a polarimeter's KDP-crystal strongly influences the Zeeman pattern, since the circular polarized parts of the two $\sigma$-components are strengthened and weakened respectively. This influence depends on the hour angle if the solar image rotates with respect to the polarizer (e.g. for Coudé telescopes).

Figure 1 schematically shows the behaviour of the Zeeman triplet Fe $\lambda 6302.5$ in
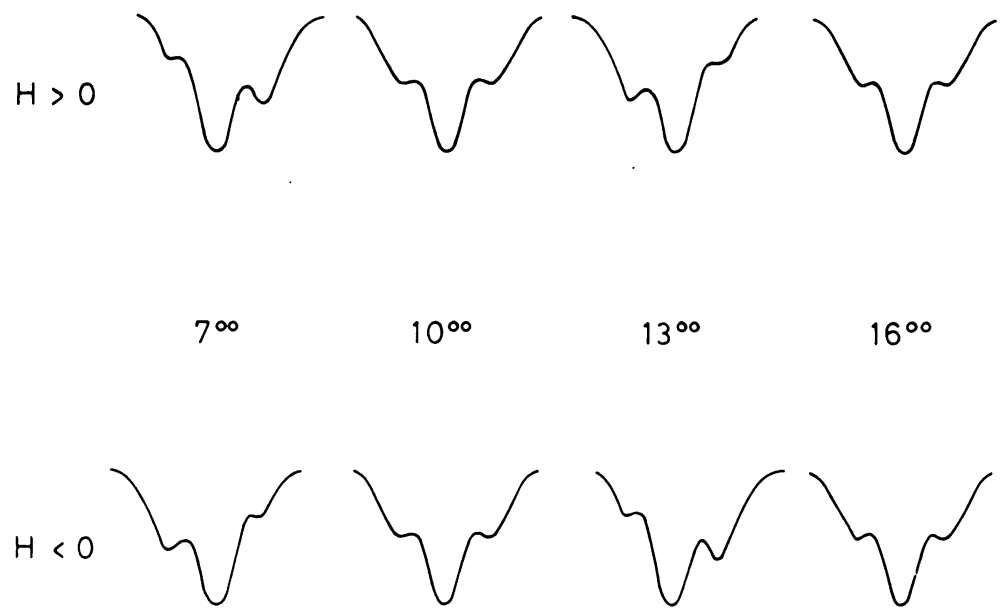

Fig. 1. Influence of the telescopic phase retardation on the Zeeman triplet Fe $\lambda 6302.5$ as measured in two sunspot umbrae of opposite polarity (schematic representation).

two sunspot umbrae of opposite polarity as measured at the Locarno observatory during one day. Since the asymmetric profiles are miscentered by the Doppler compensator, all polarimeters which separate the Stokes parameters $V$ and $U$ (or $Q$ ) using their different distribution along the line profile, cannot be used without special precautions.

In the Locarno polarimeter, for instance, which uses a three-slit arrangement, $U$ and $Q$ are measured in the $\pi$-component (Wiehr, 1969). Since $U$ and $V$ both give signals with the KDP's frequency, they can only be separated if $V$ vanishes in the central exit slit. This, however, is only assured if the inversion point $V(\lambda)=0$ coincides exactly with the center of the central slit. The above mentioned miscentering (and naturally each other miscentering!) yields a cross-talk between $U$ and $V$ which completely falsifies the sensitive $U$-signal. 
Another effect results from the decrease of circular polarization caused by the telescope's phase retardation which partially converts the circular into linear polarized light. Consequently a too small value for the quantity $V$ is measured. A comparison of measurements at $7^{\mathrm{h}}$ and $10^{\mathrm{h}}$ (see Figure 1) yields a maximal error of a factor two. A similar error occurs for the linear polarization which is diminished by the phase retardation. One thus obtains a too small value for the Stokes parameters $U$ and $Q$.

Up to now, quantitative investigations about the telescopic phase retardation have only been carried out by Jäger and Oetken (1963) for a Coelostat and for a Cassegain telescope as well as by Wiehr (1971) for the Grégory-Coudé telescope at Locarno. Similar effects are to be expected for all telescopes.

\section{References}

Jäger, F. W. and Oetken, L.: 1963, Publ. Astron. Obs. Potsdam No. 103, Vol. 31, p. 30.

Wiehr, E.: 1969, Solar Phys. 9, 225.

Wiehr, E.: 1971, Solar Phys. 18, 226. 